\author{
A. Zimpfer 1,2 \\ H. Röckmann ${ }^{1,2}$ \\ C. Zimpfer-Rechner ${ }^{1,2}$ \\ J. Utikal ${ }^{1}$ \\ E. Dippel ${ }^{1}$ \\ G. Feller ${ }^{1}$ \\ S. Goerdt ${ }^{1}$ \\ D. Schadendorf 1,2
}

\section{Neurofibromatose Typ 1 - Klinik und Genetik (Synonym: Morbus von Recklinghausen)}

\author{
Neurofibromatosis Type 1 - Clinics and Genetics
}

\section{Zusammenfassung}

Die Neurofibromatose Typ 1 ist eine der häufigsten hereditären neurokutanen Erkrankungen, die wie auch ihre klinisch bekannten Varianten autosomal dominant vererbt wird. Die Diagnose beruht auf sieben Konsensus-Kriterien des National Institute of Health (1988). Wir berichten im Folgenden über 2 Patientinnen, die mit typischen kutanen, okulären und ossären Symptomen in unserer Klinik erstmals an Neurofibromatose Typ 1 erkrankt diagnostiziert wurden. Die Klinik, die diagnostischen Kriterien sowie der genetische Hintergrund der Neurofibromatose Typ 1 sind im Folgenden dargestellt.

\section{Abstract}

Neurofibromatosis is one of most common inherited autosomal dominant neurocutaneous diseases which displays certain typical clinical characteristics. The diagnosis is based on seven criteria set up the National Institutes of Health Consensus Development Conference (1988). We describe in the following two cases of neurofibromatosis type 1 which were first diagnosed in our clinic. Both patients had the cutaneous, ocular and osseous symptoms which are typical for this disease. In this report we present the clinical aspects, the diagnostic criteria as well the genetic backround of neurofibromatosis type 1 .

\section{Einleitung}

Die Neurofibromatose ist eine der häufigsten neurokutanen Erkrankungen und stellt einen Sammelbegriff für mindestens 8 klinisch differenzierte Erscheinungsformen dar, die allerdings durchweg autosomal dominant vererbt werden [1]. Die Neurofibromatose Typ 1 (NF1) und die Neurofibromatose Typ 2 (NF2) machen dabei $95 \%$ der Neurofibromatoseformen aus [2,3].

Das verantwortliche Gen der NF1, das für das Protein Neurofibromin 1 kodiert, ist auf dem Chromosom 17 lokalisiert. Bei den zugrunde liegenden Mutationen handelt es sich um verschiedene molekulargenetische und seltener strukturelle chromosomale
Aberrationen [4]. Die NF2 ist u.a. über die Diagnose bilateraler Akustikusneurinome von der NF1 zu trennen. Das für die NF2 ursächlich verantwortliche Gen bildet das Protein Schwannomin (synonym: Merlin) und liegt auf dem Chromosom 22 [5-7]. Obwohl beide Proteine, das Neurofibromin sowie das Merlin, weder funktionelle noch strukturelle Verwandtschaft aufweisen [3], beruhen die klinischen Manifestationen der Erkrankungen sowohl bei der NF1 als auch bei der NF2 auf der fehlenden Funktion der mutierten Gene als Tumorsuppressorgene. Bei beiden Krankheiten erfolgt über den Wegfall der Hemmung nachgeschalteter Signalwege eine alterierte Stimulierung des Zellwachstums und der Zelldifferenzierung [8,9]. 

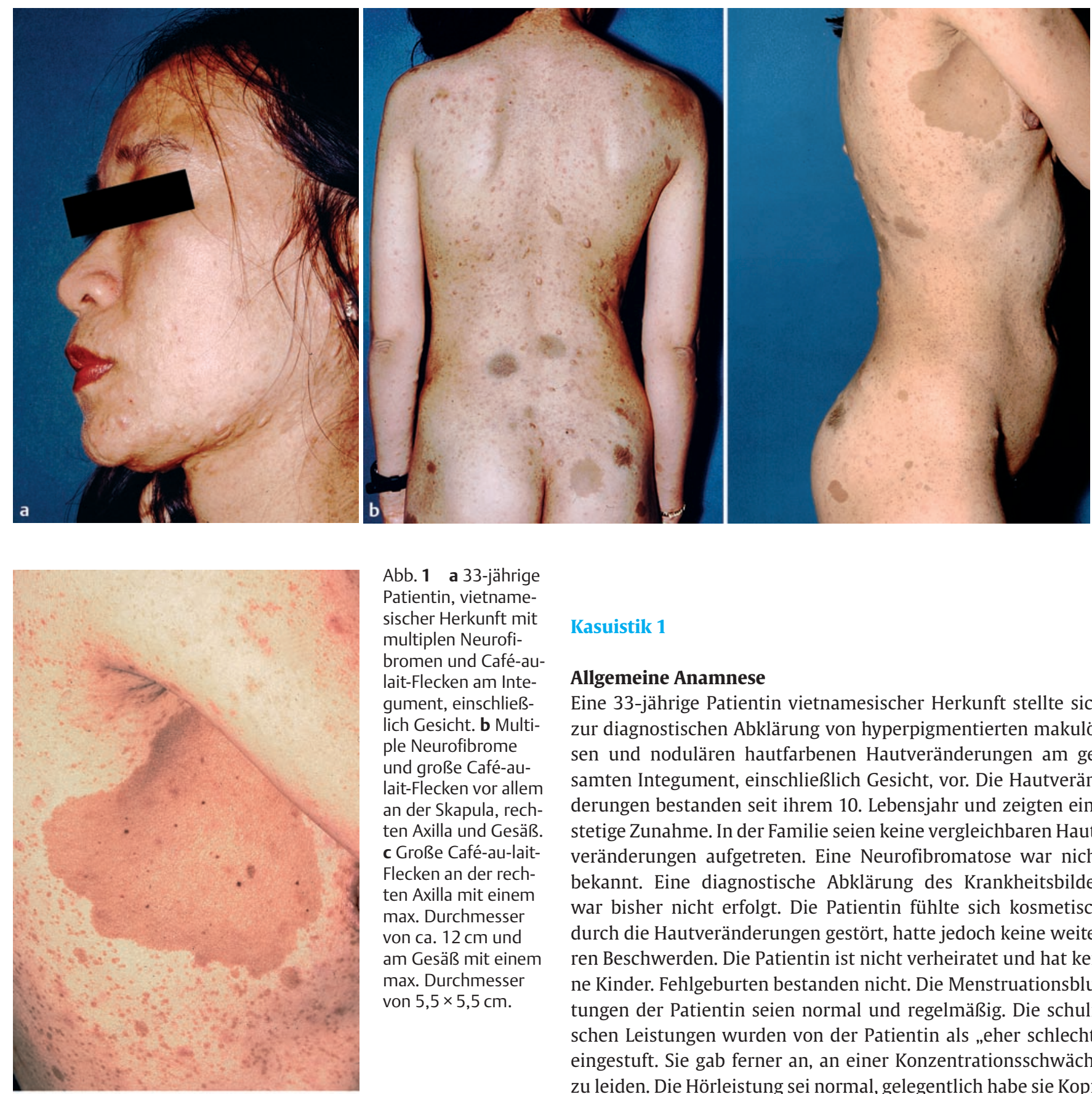

Abb. 1 a 33-jährige Patientin, vietnamesischer Herkunft mit multiplen Neurofibromen und Café-aulait-Flecken am Integument, einschließlich Gesicht. b Multiple Neurofibrome und große Café-aulait-Flecken vor allem an der Skapula, rechten Axilla und Gesäß. c Große Café-au-laitFlecken an der rechten Axilla mit einem max. Durchmesser von ca. $12 \mathrm{~cm}$ und am Gesäß mit einem max. Durchmesser von $5,5 \times 5,5 \mathrm{~cm}$.

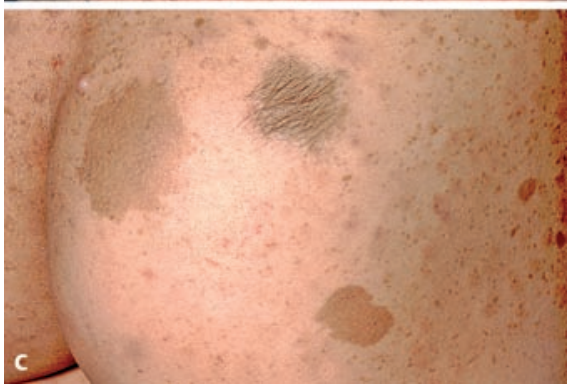

Kasuistik 1

\section{Allgemeine Anamnese}

Eine 33-jährige Patientin vietnamesischer Herkunft stellte sich zur diagnostischen Abklärung von hyperpigmentierten makulösen und nodulären hautfarbenen Hautveränderungen am gesamten Integument, einschließlich Gesicht, vor. Die Hautveränderungen bestanden seit ihrem 10 . Lebensjahr und zeigten eine stetige Zunahme. In der Familie seien keine vergleichbaren Hautveränderungen aufgetreten. Eine Neurofibromatose war nicht bekannt. Eine diagnostische Abklärung des Krankheitsbildes war bisher nicht erfolgt. Die Patientin fühlte sich kosmetisch durch die Hautveränderungen gestört, hatte jedoch keine weiteren Beschwerden. Die Patientin ist nicht verheiratet und hat keine Kinder. Fehlgeburten bestanden nicht. Die Menstruationsblutungen der Patientin seien normal und regelmäßig. Die schulischen Leistungen wurden von der Patientin als „eher schlecht“ eingestuft. Sie gab ferner an, an einer Konzentrationsschwäche zu leiden. Die Hörleistung sei normal, gelegentlich habe sie Kopfschmerzen und Schwindel. Knochen- und Gelenkbeschwerden, Sensibilitäts-, Motorik- oder Geschmacksstörungen bestanden nicht.

\section{Untersuchungsbefunde Körperliche Untersuchung}

Es befanden sich generalisiert am gesamten Integument multiple scharf begrenzte hautfarbene Papeln und Knoten mit einem max. Durchmesser von ca. $5 \mathrm{~cm}$. Eindrucksvoll zeigten sich 16 grosse Café-au-lait-Flecken mit einem Durchmesser von $>5 \mathrm{~cm}$ (rechte Skapula $9 \times 6 \mathrm{~cm}$, rechte Axilla $12 \times 9 \mathrm{~cm}$ und Gesäß

Nachfolgend beschreiben wir die klinische Präsentation von 2 Patientinnen mit Neurofibromatose Typ 1 und fassen die Kriterien für die Diagnosestellung sowie die genetischen Grundlagen der Erkrankung zusammen. $5,5 \times 5,5 \mathrm{~cm})$ sowie multiple weitere kleinere Café-au-lait-Flecken. An Rumpf und Gesicht befanden sich multiple Lentigines (Abb.1a-c). 


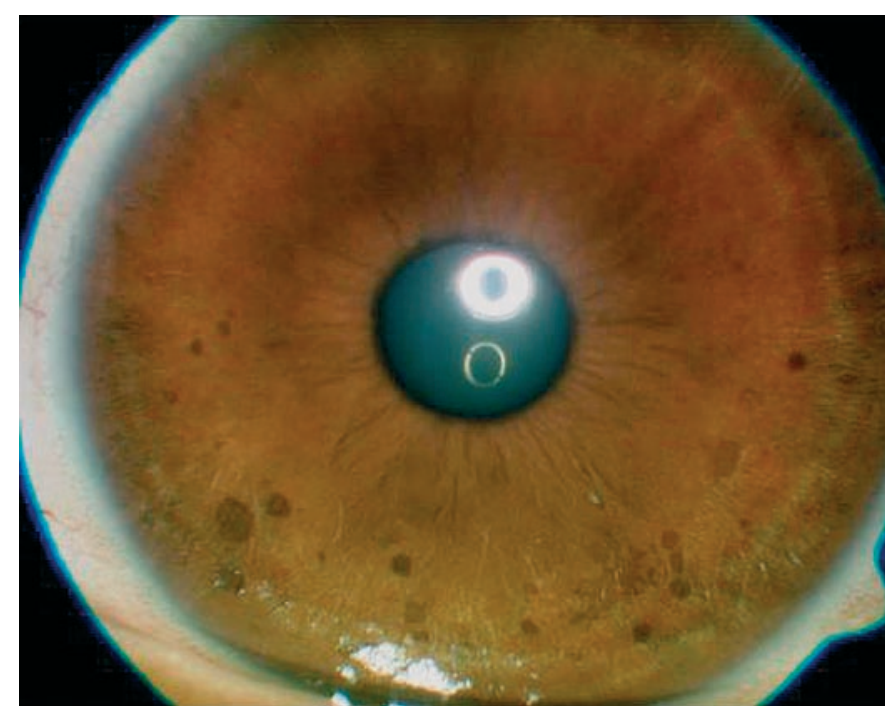

Abb. 2 Multiple scharf begrenzte bräunliche Makulae auf der Iris, so genannte Lischknötchen.

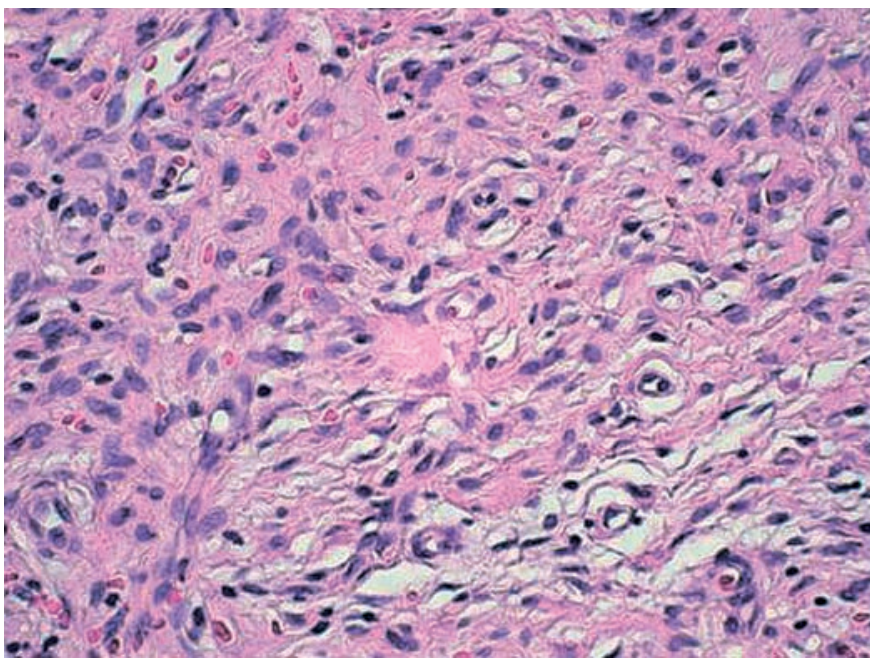

Abb. 3 Histologie der Neurofibrome: Typische zwiebelschartige Lagerung der Zellen und Zellen mit kommaförmigen Zellkernen.

\section{Ophthalmologische Untersuchung}

In der ophthalmologischen Spaltlampenuntersuchung zeigten sich multiple Lischknötchen in der Iris (Abb. 2).

\section{Histologie}

Es wurden vier der nodulären Hautveränderungen histologisch untersucht. Hier zeigten sich unscharf begrenzte Spindelzell-Läsionen, aufgebaut aus Zellen mit kommaförmigen Zellkernen. Die Zellen zeigten eine zwiebelschalenartige Lagerung mit eingestreuten Kollagenfasern, die überkleidende Epidermis keine Dysplasien.

Das histologische Bild entsprach Neurofibromen (Abb. 3).

\section{Apparative Diagnostik}

Die weitere apparative Diagnostik (Sonografie-Abdomen, Röntgen-Thorax, Röntgen-Nasennebenhöhlen, MRT-Schädel), die gynäkologische sowie neurologische Beurteilung waren ohne pa- thologischen Befund. Eine kardiologische Abklärung (Echokardiographie, EKG) erfolgte zur Abklärung eines Hypertonus aufgrund von renovaskulärer Beteiligung und zur differenzialdiagnostischen Abgrenzung eines LEOPARD-Syndroms (Lentiginosis, EKG-Veränderungen, okulärer Hypertelorismus, Pulmonalstenose, abnormale Genitalien, retardiertes Wachstum, Taubheit [deafness]).

Die internistische und die neurologische Untersuchung waren unauffällig.

\section{Kasuistik 2}

\section{Allgemeine Anamnese}

Eine 39-jährige kleinwüchsige Patientin polnischer Herkunft stellte sich zur diagnostischen Abklärung von hyperpigmentierten makulösen und nodulären hautfarbenen Hautveränderungen am gesamten Integument, einschließlich Gesicht, vor. Die Hyperpigmentierungen seien erst seit dem 12. Lebensjahr auffällig geworden. Die Neurofibrome im Gesicht und am gesamten Integument seien aber erst im Erwachsenenalter aufgetreten und nähmen an Zahl und Größe immer noch zu. Die Patientin fühlte sich kosmetisch durch die Hautveränderungen gestört. In der Familie war eine Neurofibromatose nicht bekannt. Die Hände und Füße wirkten sehr klein, wobei die Endglieder vor allem kurz waren. Die kleinen Finger zeigten eine Klinodaktylie und die zweite rechte Zehe wirkte verdickt. Die Gesichtszüge waren vergröbert, der Kopfumfang wirkte im Verhältnis zur Körpergröße groß. Die Patientin berichtete weiterhin über Sensibilitätsstörungen in den Armen und Händen und über gelegentlichen Schläfenklopfschmerz. Die Menstruationsblutungen der Patientin seien normal und regelmäßig. Knochen- und Gelenkbeschwerden, Motorik- oder Geschmacksstörungen bestanden nicht. Die Patientin ist nicht verheiratet, hat keine Kinder und lebt bei ihrer Mutter. Die Patientin hatte die Sonderschule besucht und keine Berufsausbildung abgeschlossen. Eine diagnostische Abklärung des Krankheitsbildes war bisher nicht erfolgt.

\section{Untersuchungsbefunde \\ Körperliche Untersuchung}

Es befanden sich generalisiert am gesamten Integument multiple scharf begrenzte hautfarbene Papeln und Knoten mit einem max. Durchmesser von bis zu $3 \mathrm{~cm}$. Es zeigten sich drei große Café-au-lait-Flecken mit einem max. Durchmesser von bis zu $4 \mathrm{~cm}$ (linke Flanke, rechte Skapula, Abdomen) sowie neun weitere kleinere Café-au-lait-Flecken mit einem max. Durchmesser von ca. $2 \mathrm{~cm}$. „Freckling“ fand sich in beiden Axillen (Abb. 4a,b).

\section{Ophthalmologische Untersuchung}

In der ophthalmologischen Untersuchung zeigte sich ein Lischknoten an der rechten Iris und ein Glaukom am linken Auge.

\section{Histologie}

Es wurden zehn der nodulären Hautveränderungen histologisch untersucht.

Das histologische Bild entsprach Neurofibromen. 

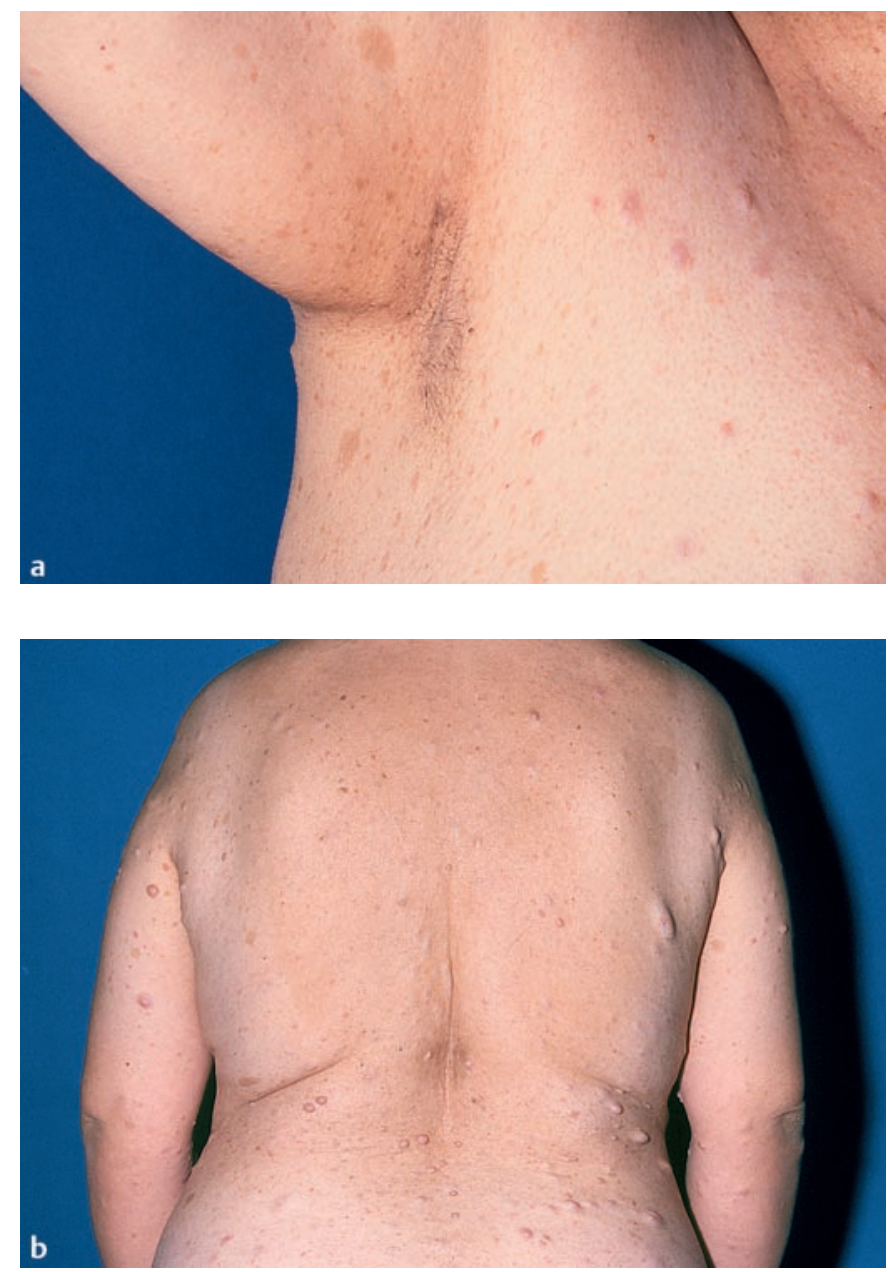

Abb. 4 a "Freckling“ (sommersprossenartige Hyperpigmentierungen) in der rechten Axilla. b Darstellung multipler Neurofibrome und einer BWS-Skoliose einer 39-jährigen Patientin.

\section{Apparative Diagnostik}

In der Sonografie des Abdomens wurden eine asymptomatische Cholezystolithiasis und eine walzenförmige Wandverdickung der Blase festgestellt. Die Röntgen-Untersuchung von Hals-, Brust- und Lendenwirbelsäule ergab eine S-förmige HWS- und BWS-Skoliose, die auch klinisch sichtbar war (Abb. 4b), sowie den hochgradigen Verdacht auf Sanduhrneurinome im Bereich der HWK 4 bis BWK 1.

In der neurologischen Untersuchung wurden eine linksseitige leichtgradige Parese der kleinen Handmuskeln und eine Hyperalgesie und Dysästhesie im Dermatom C7/C8 diagnostiziert. Die HNO-ärztliche Untersuchung stellte die Verdachtsdiagnose einer retrokochleären Hörstörung bei unauffälligem MRT-Schädel-Befund. Die gynäkologische und urologische Beurteilung (mit Zystoskopie) waren ohne pathologischen Befund. Die internistische Untersuchung war unauffällig.

\section{Genetische Untersuchung}

Karyogramm: Es fand sich ein regelrechter weiblicher Chromosomensatz, $46 \mathrm{XX}$, ohne strukturelle Veränderungen und ohne Hinweis auf eine mikroskopisch sichtbare Deletion im Chromosom 17.
Tab. 1 Zur Diagnosestellung einer Neurofibromatose Typ 1 müssen zwei der sieben Konsensus-Kriterien des National Institute of Health von 1988 erfüllt sein [12]

$\geq 6$ Café-au-lait-Flecken (präpubertär: $>\varnothing 0,5 \mathrm{~cm}$, postpubertär: $>\varnothing 1,5 \mathrm{~cm}$
Optikusgliom
$\geq 2$ Lischknötchen Irishamartome
Knochenläsionen, Keilbeindysplasie, Ausdünnung der Röhrenknochen
(mit oder ohne Pseudoarthrose)
„Freckling“ inguinal/axillär
2 oder mehrere subkutane Neurofibrome oder 1 plexiformes Neurofibrom
betroffener Verwandter I. Grades

Tab. 2 Einteilung der verschiedenen klinischen Erkrankungsformen der Neurofibromatose nach Riccardi $[1,10]$

\begin{tabular}{ll}
\hline NF Typ I: Neurofibromatose von Recklinghausen & (NF 1) \\
\hline NF Typ II: Bilaterale Akustikusneurofibromatose & (NF 2) \\
\hline Gemischte Form: NF 1 und NF 2, multiple ZNS-Tumoren & (NF 3) \\
\hline Diffuse Café-au-lait-Flecken (CLF) und große Neurofibrome & (NF 4) \\
\hline Segmentale CLF, Neurofibrome & (NF 5) \\
\hline Diffuse CLF, keine Neurofibrome, geistige Retardierung & (NF 6) \\
\hline Late onset-NF: 3. Dekade, tiefe Neurofibrome & (NF 7) \\
\hline Unspezifische NF & (NF 8) \\
\hline
\end{tabular}

\section{Besprechung beider Kasuistiken}

Die Diagnose einer NF Typ 1 wird aufgrund der derzeit gültigen 1988 verabschiedeten Konsensus-Kriterien des National Institute of Health gestellt, die in Tab.1 zusammengefasst sind. Unsere erste Patientin wies drei der sieben Kriterien einer NF1 auf: mehr als 5 große $(>10 \mathrm{~cm}$ ) Café-au-lait-Flecken, Neurofibrome, Lischknoten. Eine systemische Beteiligung wurde nicht festgestellt.

Die zweite Patienten wies vier der sieben Kriterien auf: mehr als 6 Café-au-lait-Flecken (max. Durchmesser zwischen 2-4cm), Neurofibrome und plexiforme Neurofibrome, eine S-Skoliose und axilläres „Freckling“. Die mechanische Einengung von Austrittspunkten peripherer Nerven z.B. durch Sanduhrneurinome führt oftmals zu neurologischen Ausfällen wie auch bei unserer Patientin mit Handmuskelatrophien und Parästhesien.

\section{Diskussion}

Die Neurofibromatose ist eine der häufigsten genetischen Erkrankungen in der Dermatologie. Nach Riccardi werden acht verschiedene klinische Bilder (Tab. 2 ) unterschieden $[1,10]$, wobei die Neurofibromatose Typ 1 mit einer Prävalenz von 1:3500-5000 die häufigste Erscheinungsform ist. Die Neurofibromatose Typ 2 ist um das 10-20fache seltener. Die weiteren sechs bekannten Formen der Neurofibromatose betreffen zusammen nur ca. 5\% der Neurofibromatose-Patienten und sind somit Raritäten $[10,11]$. 
Die typischen klinischen Merkmale der Neurofibromatose Typ 1 sind Café-au-lait-Flecken, sommersprossenartige Hyperpigmentierungen (Freckling) in den Axillen und Leisten, Lischknoten an der Iris, Neurofibrome, Knochenveränderungen und Optikusgliome. Die Café-au-lait-Flecken treten in über $90 \%$ der Fälle schon vor dem zweiten Lebensjahr auf, während sich die Neurofibrome oft erst nach der Pubertät entwickeln. Zu der Krankheit können auch eine vergleichsweise geringe Körpergröße und ein relativ großer Kopfumfang - wie bei unserer zweiten Patientin - gehören. Zur Diagnosestellung einer Neurofibromatose Typ 1 wurden 1988 sieben Konsensus-Kriterien des National Institute of Health (Tab.1) festgelegt. Zwei davon müssen definitionsgemäß zur Diagnosestellung einer Neurofibromatose Typ 1 erfüllt sein [12]. Die Neurofibromatose Typ 2 wird durch bilaterale Akustikusneurinome von der Neurofibromatose Typ 1 unterschieden. Beide Erkrankungen werden als Keimbahnmutation in Familien autosomal dominant vererbt [13], Neumutationen sind häufig [14].

Wenn bei den Eltern keine Symptome einer Neurofibromatose gefunden werden, wie zum Beispiel bei unseren beiden Patientinnen, so muss es sich um eine Neumutation handeln, d.h. die Veränderung ist in der Keimzelle eines Elternteils aufgetreten, so dass sich die Erkrankung erstmals bei den Kindern manifestieren kann. Der molekulargenetische Nachweis einer Mutation ist im Neurofibromatose-Gen möglich, doch wegen der Größe des Gens (60 Exons) und der enormen Anzahl verschiedener Mutationen sehr aufwändig und kostspielig. Eine Mutation kann ca. bei $70 \%$ aller Betroffenen nachgewiesen werden. Auch wenn keine Mutation gefunden wird, ist die Erkrankung dadurch nicht ausgeschlossen. Die Art der Mutation lässt keine Rückschlüsse auf den weiteren Verlauf der Erkrankung zu, so dass im Allgemeinen eine molekulargenetische Untersuchung nicht indiziert ist.

Das Schlüsselprotein der Neurofibromatose Typ 1 (Neurofibro$\min 1$ ) hat eine Tumorsuppressorfunktion [9]. Das entsprechende Gen findet sich auf dem langen Arm des Chromosoms 17. Das Genprodukt ist in allen Gewebszellen vorhanden, wird aber in neuroektodermalen Zellen besonders hoch exprimiert [2]. Neurofibromin 1 ist durch die Bindung seiner „GTPase activating Protein" (GAP)-Domäne an das Protoonkogenprodukt p21RAS in der Lage, das gebundene GTP zu hydrolysieren, p21RAS wird somit in die inaktive Form überführt. Dies führt wiederum zur Reduzierung von Zellwachstum und Zelldifferenzierung. Umgekehrt verliert bei einem Gendefekt das funktionell fehlgesteuerte oder fehlende Neurofibromin 1 seine hemmende Wirkung auf p21RAS, woraus eine Stimulierung von Zellwachstum und Zelldifferenzierung resultiert $[8,9,15]$ (Abb. 5).

Komplikationen, die vermehrt beobachtet werden, sind z.B. Malignome, Wirbelsäulenverkrümmung, Glaukome, Stenosierung des Spinalkanals, Pseudoarthrosen, Skoliosen oder eine Lernbehinderung. Eine wichtige Komplikation ist das so genannte Optikusgliom, das jedoch in der Regel vor dem 20. Lebensjahr auftritt. Glaukome können durch periorbitale plexiforme Neurofibrome verursacht werden. Paravertebrale Neurofibrome („Sanduhrneurinome“) können in den Spinalkanal eindringen und im Folgenden zur Querschnittsymptomatik führen. Wegen der zahlreichen möglichen Komplikationen der Erkrankung ist die frühzeitige Diagnosestellung der Neurofibromatose wichtig. Hierbei

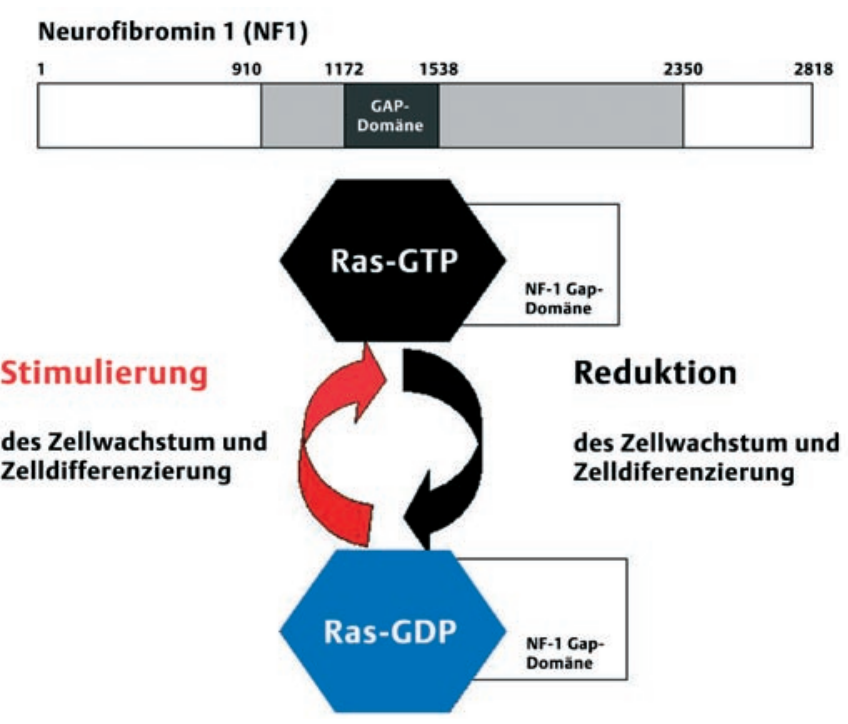

Abb. 5 Darstellung des vermuteten Wirkmechanismus des mutierten Neurofibromin 1: Das Neurofibromin 1 soll durch die Bindung seiner GAP-Domäne an das Protoonkogenprodukt p21RAS in der Lage sein, dieses in eine inaktive Form zu überführen. Dies führt zur Reduzierung von Zellwachstum und Zelldifferenzierung. Umgekehrt führt die Mutation des Neurofibromin 1 bei der Neurofibromatose Typ 1 zu einer Stimulierung des Zellwachstums und der Zelldifferenzierung.

ist das altersabhängige Auftreten der Komplikationen zu berücksichtigen: Die Malignominzidenz ist bei der Neurofibromatose im Vergleich zu der Allgemeinbevölkerung deutlich erhöht und steigt mit dem Alter. Die Tumorsuche sowie frühzeitiges Erkennen von begleitenden Komplikationen der Erkrankung sollte durch jährliche dermatologische, neurologische, ophthalmologische, orthopädische, internistische und psychologische Untersuchung erfolgen. Weitere Diagnostik sollte symptomorientiert durchgeführt werden. Eine ausführliche genetische Beratung mit Informationen zu der Erkrankung ist sowohl für die Patienten als auch für die Familienangehörigen empfehlenswert.

Die Therapie ist rein symptomatisch. Das häufigste Problem sind kosmetisch störende Hautveränderungen, vor allem die Neurofibrome, die mittels Laser abladiert werden oder operativ exzidiert werden können. Lernschwierigkeiten, deren Ursache unbekannt ist, sind bei $30 \%$ der betroffenen Kinder vorhanden, so dass eine entsprechende Förderung wichtig ist. Pseudoarthrosen oder Kyphoskoliosen sollten frühzeitig operativ korrigiert werden.

Die Neurofibromatose kann momentan noch nicht kausal behandelt werden. Pharmakotherapeutisch einsetzbare Modulatoren der mutierten Tumorsuppressoren oder nachgeschalteter Onkogene könnten möglicherweise die Inzidenz von bösartigen Tumoren bei Neurofibromatose-Patienten senken.

\section{Literatur}

${ }^{1}$ Riccardi VM. The clinical and molecular genetics of Neurofibromatosis-1 and Neurofibromatosis-2. In: Rosenberg RN, Prusiner SB, DiMauro $S$ et al. (eds). The molecular and genetic basis of neurological disease. Boston: Butterworth-Heinemann, 1993: 837-853

2 Colman SD, Wallace MR. Neurofibromatosis type 1. Eur J Cancer 1994; 30: $1974-1981$ 
${ }^{3}$ Thomas G, Mérel P, Sanson M et al. Neurofibromatosis type 2. Eur J Cancer 1994; 30 A: 1981 - 1987

${ }^{4}$ Schmidt MA, Michels VV, Dedwald GW. Cases of neurofibromatosis with rearrangements of chromosome 17 involving band 17q11.2. Am J Hum Genet 1987; 28: $771-777$

${ }^{5}$ Morrison H, Sherman LS, Legg J et al. The NF2 tumor suppressor gene product, merlin, mediates contact inhibition of growth through interactions with CD44. Genes Dev 2001; 15: 968 - 980

${ }^{6}$ Sun C-X, Robb VA, Gutmann DH. Protein 4.1 tumor suppressors: getting a FERM grip on growth regulation. J Cell Science 2002; 115: 3991 4000

${ }^{7}$ Trofatter JA, MacCollin MM, Rutter JL et al. A novel moesin-, ezrin-, radixin-like gene is a candidate for the neurofibromatosis 2 tumor suppressor. Cell 1993; 72: 1 - 20

8 Cichowski K, Jacks T. NF1 tumor supressor gene function: narrowing the gap. Cell 2001; 105: 593-604

${ }^{9}$ McCormick F. Ras signaling and NF1. Curr Opinion Genes Dev 1995; 5 : $51-55$

${ }^{10}$ Riccardi VM. The multiple forms of neurofibromatosis. N Engl J Med 1982; 305: $1617-1627$

${ }^{11}$ Jung EG. Segmental Neurofibromatosis (NF5). Neurofibromatosis, 1988; 1 : $306-311$

12 National Institutes of Health Consensus Developement Conference. Neurofibromatosis: conference statement. Arch Neurol 1988; 45: $575-578$

${ }^{13}$ Lázaro C, Ravenna A, Gaona A, Volpini V, Estivill X. Neurofibromatosis type 1 due to germ - line mosaicism in a clinically normal father. $\mathrm{N}$ Engl J Med 1994; 331: $403-1407$

${ }^{14}$ Köhler B. Neurofibromatose im Kindesalter. Stuttgart: Wiss. Verlagsgesellschaft, 1990

15 Ridley AJ, Paterson HF, Noble M, Land H. Ras - mediated cell cycle arrest is altered by nuclear oncogenes to induce Schwann cell transformation. EMBO J 1988; 7: 1635-1645

\section{Preisausschreibung}

\section{Hans Dengler Forschungsstipendium für Klinische Pharmakologie}

Die Universität Heidelberg schreibt im Jahr 2004 zum sechten Mal das Hans Dengler Forschungsstipendium für Klinische Pharmakologie aus. Das Stipendium ist mit einem Betrag in Höhe von $€ \mathbf{8 0 0 0 , -}$ dotiert.

Junge, promovierte Wissenschaftler, die ein Höchstalter von 35 Jahren noch nicht überschritten haben, sind eingeladen, sich mit einem Projekt mit Bezug zur Klinischen Forschung schriftlich zu bewerben.

Die Bewerbungsunterlagen sollten enthalten:

- Lebenslauf

- Publikationsverzeichnis

- Projektskizze, max. 3 Seiten

- zwei Referenzanschriften

Die eingehenden Bewerbungen werden von einer fachkompetenten Jury beurteilt. Bewerbungen werden bis zum 1. Oktober $\mathbf{2 0 0 4}$ erbeten an das

Rektorat der Universität Heidelberg Herrn Prorektor Prof. Dr. Jochen Tröger Postfach 105760

69047 Heidelberg 